ESAIM: PROCEEDINGS, October 2007, Vol. 22, 181-189

Gabriel Caloz \& Monique Dauge, Editors

\title{
CALCULS DE SENSIBILITE PAR DIFFERENTIATION POUR L'AÉRODYNAMIQUE
}

\author{
Alain Dervieux ${ }^{1}$, Youssef Mesri $^{2}$, Francois Courty ${ }^{1}$, Laurent Hascoet $^{1}$, \\ Bruno Koobus ${ }^{3}$ and Mariano VAzQuez ${ }^{4}$
}

\begin{abstract}
Both continuous and discrete sensititivites and gradient evaluations carry important information in the building of an optimal shape loop. This is particularly true for the smoothness of objective functionals and the functions representing their gradients. The main application addressed here is the choice of a preconditioner for the optimisation loop.

Résumé. Gradient continu, gradient discret, tous deux nous apportent des informations importantes pour les boucles de conception optimale de forme, notamment en ce qui concerne la régularité des fonctionnelles et des fonctions constituées par leur gradients. La principale application présentée est le choix d'un préconditionneur pour la boucle d'optimisation.
\end{abstract}

\section{INTRODUCTION}

L'application des méthodes du Contrôle Optimal pour la conception de formes optimales en Aéronautique est de plus en plus répandue. Des gradients de fonctionnelles sont construits efficacement en calculant des états adjoints. Les premières applications en Aéronautique ont relancé le débat entre tenants du gradient continu discrétisé et tenants du gradient de la fonctionnelle discrète. Voir par exemple [14,19,20] . L'utilisation de gradients continus discrétisés pose le problème de leur convergence vers le discret et donc le problème de la régularité du gradient en tant que fonction. L'utilisation directe de gradients de la fonctionnelle discrète tend à masquer les aspects fonctionnels sous-jacents aussi bien que la question primordiale de la différentiabilité de la fonctionnelle dont on prétend manipuler le gradient. On se propose ici de rassembler quelques questions de régularité dans le contexte fonctionnel: quelles sont la différentiabilité de la fonctionnelle, dans quels espaces, la régularité de la fonction gradient notamment en présence de choc, quelles sont les conséquences algorithmiques de ces propriétés de régularités. Ce papier synthétise et prolonge les réflexions sur ce sujet développées par les co-auteurs dans les papiers cités en bibliographie.

${ }^{1}$ INRIA Tropics Project-Team, 2004 route des lucioles, BP 93, 06902 Sophia-Antipolis, FRANCE (www-sop.inria.fr/tropics)

2 INRIA SMAsh Project-Team, 2004 route des lucioles, BP 93, 06902 Sophia-Antipolis, FRANCE (www-sop.inria.fr/smash)

3 ACSCIOM, Département de Mathématiques, Université de Montpellier II, Case Courrier 51, 34095 Montpellier Cedex 5, France. koobus@darboux.math.univ-montp2.fr

${ }^{4}$ Computational Science and Mechanics Team, Barcelona Supercomputing Center, Nexus II - Campus Nord UPC C/ Jordi Girona, 29, 08034 Barcelona - Spain (http://www.bsc.es/csmteam)

(C) EDP Sciences, SMAI 2007 


\section{Gradient en COntinu}

Les questions centrales de ce paragraphe sont: la fonctionnelle est-elle différentiable, à partir de quel espace fonctionnel, quelle est la régularité de la fonction représentant sa dérivée, dans quels espaces est-elle un opérateur linéaire borné? Le calcul des variations de la solution d'une Equation aux Dérivées Partielles par rapport à son domaine a été abordé il y a plus d'un siècle par Hadamard, et a connu un regain de faveur après son redémarrage sous l'impulsion de Jean Céa. Une difficulté théorique majeure pour la construction d'une méthode de gradient réside dans la difficulté à introduire un espace de Hilbert optimal pour le paramétrage du domaine. Nous nous contentons ici d'un paramétrage optimal dans des espaces de Banach.

\subsection{Problème modèle}

Soit $\Omega_{0}$ un domaine géométrique régulier de $R^{d}(\mathrm{~d}=2$ ou 3$)$. On paramétrise un ensemble de perturbations $\Omega_{\gamma}$ de $\Omega_{0}$ toutes comprises dans un sur-domaine commun $\mathcal{O}$ et comprenant un sous-domaine commun $D$. Cela est réalisé en considérant des fonctions $\gamma$ de $\mathcal{C}^{l+\alpha}\left(\partial \Omega_{0}\right)$ et en déplaçant chaque point $\vec{x}$ de $\partial \Omega_{0}$ suivant la normale $\vec{n}_{0}$ à $\partial \Omega_{0}$ d'une distance $\gamma(\vec{x})$ :

$$
\forall \gamma \in \mathcal{C}^{l+\alpha}\left(\partial \Omega_{0}\right): \quad \Omega_{\gamma} \subset \mathcal{O} \quad ; \quad D \subset \Omega_{\gamma} \quad ; \quad \partial \Omega_{\gamma}=\left\{\vec{x}+\gamma(\vec{x}) \vec{n}_{0}, \vec{x} \in \partial \Omega_{0}\right\} .
$$

Soit $z(\gamma)$ la solution du problème de Dirichlet homogène avec source $f$ dans le domaine $\Omega_{\gamma}$ :

$$
-\Delta z(\gamma)=f \operatorname{sur} \Omega_{\gamma} ; z(\gamma)=0 \text { sur } \partial \Omega_{\gamma}
$$

On cherche le paramètre de forme $\gamma$ qui minimisera une fonctionnelle à valeurs réelles $j$ ( $z_{c i b l e}$ donné):

$$
j(\gamma)=\frac{1}{2}\left\|z(\gamma)-z_{c i b l e}\right\|_{D}^{2} .
$$

En faisant les hypothèses suffisantes sur les données, on peut prolonger $z(\gamma)$ dans tout le domaine $\mathcal{O}$ (cf. par exemple [10]) et cette application est $C^{1}$ :

$$
\bar{z}: \gamma \mapsto \bar{z}(\gamma) \text { tel que }\left.\bar{z}(\gamma)\right|_{\Omega_{\gamma}}=z(\gamma) \text { est } \mathbf{C}^{1}: \mathcal{C}^{l+\alpha}\left(\partial \Omega_{0}\right) \rightarrow \mathcal{C}^{l-1+\alpha}(\mathcal{O})
$$

Il est alors possible de calculer la dérivée composée de la fonctionnelle suivante (cf. [7]):

$$
\begin{aligned}
& \Psi\left(\gamma, z ; \phi_{1}, \phi_{2}\right)=-\int_{\Omega_{\gamma}}(\Delta z-f) \phi_{1} d v+\int_{\partial \Omega_{\gamma}} z \phi_{2} d \sigma \\
& \Psi\left(\gamma, \bar{z}(\gamma) ; \phi_{1}, \phi_{2}\right)=0 \forall \phi_{1}, \forall \phi_{2} .
\end{aligned}
$$

et on en déduit la propriété suivante vérifiée par la dérivée de $\bar{z}$ :

$$
-\Delta \frac{\partial \bar{z}}{\partial \gamma}(\gamma) \cdot \delta \gamma=0 \text { dans } \Omega_{\gamma} \quad ; \quad \frac{\partial \bar{z}}{\partial \gamma}(\gamma) \cdot \delta \gamma=\frac{\partial \bar{z}(\gamma)}{\partial n_{\gamma}}\left(n_{\gamma}, n_{0}\right) \delta \gamma \quad \text { sur } \quad \partial \Omega_{\gamma}
$$

La dérivée de la fonctionnelle s'en déduit de manière classique en introduisant l'état adjoint $\pi$ solution du système adjoint:

$$
\begin{gathered}
-\Delta \pi(\gamma)=\chi_{D}\left(z_{c i b l e}-z(\gamma)\right) \text { dans } \Omega_{\gamma} ; \pi(\gamma)=0 \text { sur } \partial \Omega_{\gamma} \\
j^{\prime}(\gamma) . \delta \gamma=\int_{\partial \Omega_{\gamma}} \frac{\partial z(\gamma)}{\partial n_{\gamma}} \frac{\partial \pi(\gamma)}{\partial n_{\gamma}}<\vec{n}_{\gamma}, \vec{n}_{0}>\delta \gamma d \partial \Omega_{\gamma}
\end{gathered}
$$


On constate que, les intégrandes étant sommables, cette dérivée s'exprime comme l'application d'un gradient dans $L^{2}\left(\partial \Omega_{0}\right)$ sur l'acccroissement $\delta \gamma$ :

$$
j^{\prime}(\gamma) \cdot \delta \gamma=<g_{L^{2}}, \delta \gamma>; g_{L^{2}}(\gamma)=\frac{\partial z(\gamma)}{\partial n_{\gamma}} \frac{\partial \pi(\gamma)}{\partial n_{\gamma}}<\vec{n}_{\gamma}, \vec{n}_{0}>
$$

et il est tentant de prendre $L^{2}\left(\partial \Omega_{0}\right)$ comme espace pivot d'une méthode de gradient et $g_{L^{2}}(\gamma)$ comme direction de descente:

$$
\gamma^{*}=\gamma-\rho g_{L^{2}}(\gamma) \text {. }
$$

Ceci n'est pas possible, $g_{L^{2}}(\gamma)$ étant a priori moins régulier que $\gamma$ :

$$
g_{L^{2}}: \quad \mathcal{C}^{l+\alpha}\left(\partial \Omega_{0}\right) \rightarrow \mathcal{C}^{l-1+\alpha}\left(\partial \Omega_{0}\right),
$$

une propriété bien évidente quand on constate les dérivations des états et la présence de la normale dans l'expression de $g_{L^{2}}(\gamma)$. Itérer avec ce gradient aura pour conséquence une perte de dérivation à chaque pas jusqu'à ce que la frontière soit insuffisamment régulière pour continuer. Il est important de noter que cette perte d'un ordre de dérivation n'est pas une loi générale mais nécessite d'être évaluée cas pas cas. Dans [2], Arian et Salas exhibent des fonctionnelles "inadmissibles" comme la suivante:

$$
j(\gamma)=\frac{1}{2} \int_{\partial \Omega_{\gamma}}\left(\frac{\partial^{k} z(\gamma)}{\partial s^{k}}-z_{\text {cible }}\right)^{2} d \sigma \quad \text { avec } \quad:-\Delta z(\gamma)=f \text { sur } \Omega_{\gamma} ; z(\gamma)=0 \text { sur } \partial \Omega_{\gamma} .
$$

Dans ce cas typique, état adjoint et dérivée de la fonctionnelle s'écrivent:

$$
\begin{aligned}
-\Delta \pi(\gamma) & =0 \operatorname{sur} \Omega_{\gamma} ; \pi(\gamma)=-\frac{\partial^{k}}{\partial s^{k}}\left(\frac{\partial^{k} z(\gamma)}{\partial s^{k}}\right) \text { sur } \partial \Omega_{\gamma} . \\
g_{L^{2}} & : \quad \mathcal{C}^{l+\alpha}\left(\partial \Omega_{0}\right) \rightarrow \mathcal{C}^{l-k-1+\alpha}\left(\partial \Omega_{0}\right)
\end{aligned}
$$

ce qui met en évidence une perte de $k+1$ ordres de différentiation spatiale.

Supposons que nous disposions d'un opérateur linéaire défini positif recouvrant la perte de régularité:

$$
B \quad: \quad \mathcal{C}^{l-k-1+\alpha}\left(\partial \Omega_{0}\right) \rightarrow \mathcal{C}^{l+\alpha}\left(\partial \Omega_{0}\right) \quad ; \quad<h, B h>_{L^{2}\left(\partial \Omega_{0}\right)} \geq \epsilon\|h\|_{L^{2}\left(\partial \Omega_{0}\right)},
$$

alors $B g_{L^{2}}$ sera une direction de descente pour $j$.

\subsection{Aérodynamique: équations d'Euler}

Le cas des équations d'Euler ne permet dans l'état actuel de la théorie de réaliser une analyse rigoureuse analogue à la précédente. Néanmoins, un rapide calcul formel permet une assez bonne identification de la perte de dérivation spatiale (voir aussi $[1,5]$ ). Les équations d'Euler stationnaires sont écrites en bref comme suit:

$$
\begin{aligned}
W_{1}=\rho, W_{2}=\rho u, W_{3}=\rho v, W_{4} & =\rho w, W_{5}=E \quad ; \quad F(W)_{x}+G(W)_{y}+H(W)_{z}=0 \\
(\Psi(\gamma, W), \phi)=\quad & -\int_{\Omega_{\gamma}}\left(F(W) \cdot \frac{\partial \phi}{\partial x}+G(W) \cdot \frac{\partial \phi}{\partial y}+H(W) \cdot \frac{\partial \phi}{\partial z}\right) d \Omega_{\gamma} \\
& +\int_{\partial \Omega_{B}}\left(F_{B} n^{x}+G_{B} n^{y}+H_{B} n^{z}\right) \cdot \phi d \sigma \\
& +\int_{\partial \Omega_{\gamma}} p(W)\left(n_{x}^{\gamma} \phi_{2}+n_{y}^{\gamma} \phi_{3}+n_{z}^{\gamma} \phi_{4}\right) d \sigma=0 .
\end{aligned}
$$


Les notations $F, G, H$ sont les classiques flux des équations d'Euler et $p(W)$ désigne la pression (fonction de $W$ via la loi d'état). La deuxième intégrale concerne les conditions aux limites d'entrées et sorties et n'a pas besoin d'être précisée dans notre étude. La fonctionnelle $j(\gamma)=J(\gamma, W(\gamma))$ n'est pas toujours différentiable. On admet ici qu'elle l'est. On a:

$$
\begin{aligned}
j^{\prime}(\gamma) \cdot \delta \gamma= & <g_{L^{2}}, \delta \gamma> \\
g_{L^{2}}(\gamma, W, \Pi)=\quad & -\left(F(W) \frac{\partial \Pi}{\partial x}+G(W) \frac{\partial \Pi}{\partial y}+H(W) \frac{\partial \Pi}{\partial z}\right)\left(\vec{n}^{\gamma} \cdot \vec{V}\right) \\
+ & (\nabla p(W) \Pi+p(W) \nabla \Pi)\left(\vec{n}^{\gamma} \cdot \vec{V}\right),
\end{aligned}
$$

avec:

$$
\begin{gathered}
\left(\frac{\partial F}{\partial W}\right)^{*} \frac{\partial \Pi}{\partial x}+\left(\frac{\partial G}{\partial W}\right)^{*} \frac{\partial \Pi}{\partial y}+\left(\frac{\partial H}{\partial W}\right)^{*} \frac{\partial \Pi}{\partial z}=-\frac{\partial J}{\partial W}, \\
\Pi_{2} n_{x}^{\gamma}+\Pi_{3} n_{y}^{\gamma}+\Pi_{4} n_{z}^{\gamma}=0 \text { on } \partial \Omega_{\gamma} .
\end{gathered}
$$

À nouveau considérons l'itération de gradient pour l'espace pivot $L^{2}$ :

$$
\gamma=\gamma-\rho g_{L^{2}}(\gamma, W, \Pi)
$$

En supposant tous les champs suffisamment réguliers, nous constatons à nouveau une perte de dérivation d'un ordre. Nous discuterons au paragraphe 4 des conséquences de cette analyse. L'hypothèse de régularité des champs devient irréaliste dans le cas d'écoulements comportant des chocs. Nous abordons ce point au paragraphe suivant.

\section{3. Écoulements compressibles à champs discontinus}

Les écoulements à champs discontinus sont à l'origine de problèmes de non-différentiabilité des fonctionnelles. Dans le cas non-différentiable, il est encore possible d'utiliser des méthodes sous-gradients, cf. par exemple [18]. Bardos et Pironneau ont cependant établi la différentiabilité d'une famille de fonctionnelles lorsque l'écoulement comporte des chocs (cf. $[3,4]$ ). Même dans le cas où la fonctionnelle est différentiable, sa dérivée s'exprime en fonction de l'état et de l'état adjoint, lesquels présentent de fortes singularités. Ces singularités sont étudiées notamment par Giles et Pierce [13,15]. Concentrons nous sur un écoulement transsonique simple, l'écoulement dans une tuyère à section presque constante (figures 1 et 2). L'étude de Giles et Pierce [15] propose une solution analytique du cas limite quasi 1D et permettent d'identifier les singularités présentes dans les états.

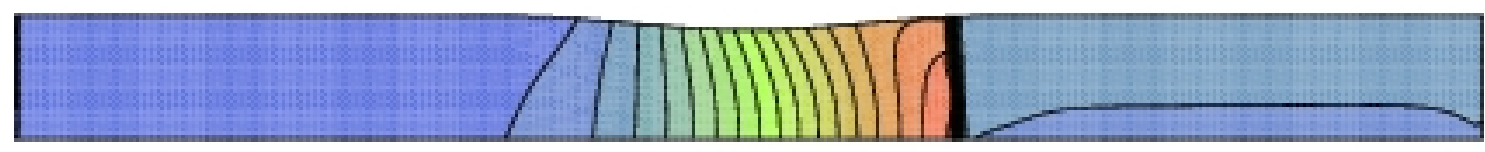

Figure 1: Optimisation d'une tuyère en transsonique. Etat: nombre de Mach

Si les champs de l'état comportent des chocs (du supersonique vers le subsonique), ceux de l'état adjoint montrent des points de rebroussements dans les passages du subsonique vers le supersonique. Dans la figure 1 on reconnait le choc quasi-vertical vers la fin (à droite) du rétrécissement, tandis que dans la figure 2 qui présente la deuxième composante $\Pi_{2}$ de l'état adjoint, on distingue au premier tiers du rétrécissement une structure verticale à peine plus épaisse. Le gradient (5) s'exprime en fonction des états et de leur dérivées premières. Il doit en résulter pour cette dérivée une discontinuité au niveau de la ligne sonique et une couche de Dirac sur le choc. Dans ce cas, les gradients continus discrétisés peuvent poser des problèmes d'approximation. Inversement, on peut se demander si les gradients discrets conservent quelque souvenir du gradient continu dont ils constituent des discrétisations (pas forcément convergentes). Nous présentons en figure 3 une série de 
gradient $g_{L^{2}}$ discrets pour des maillages emboités de plus en plus fins. On constate une certaine cohérence mais pas vraiment de convergence numérique. Il semble donc plus sûr de disposer de gradients de la fonctionnelle discrète si le but à court terme est de minimiser cette fonctionnelle. Nous abordons maintenant l'étude des techniques permettant la dérivation discrète.

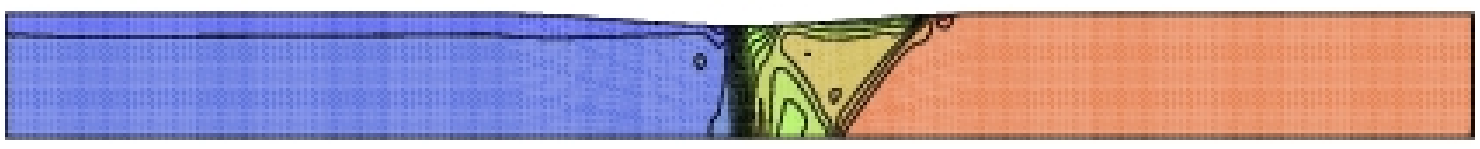

Figure 2: Optimisation d'une tuyère en transsonique. Etat adjoint, deuxième composante

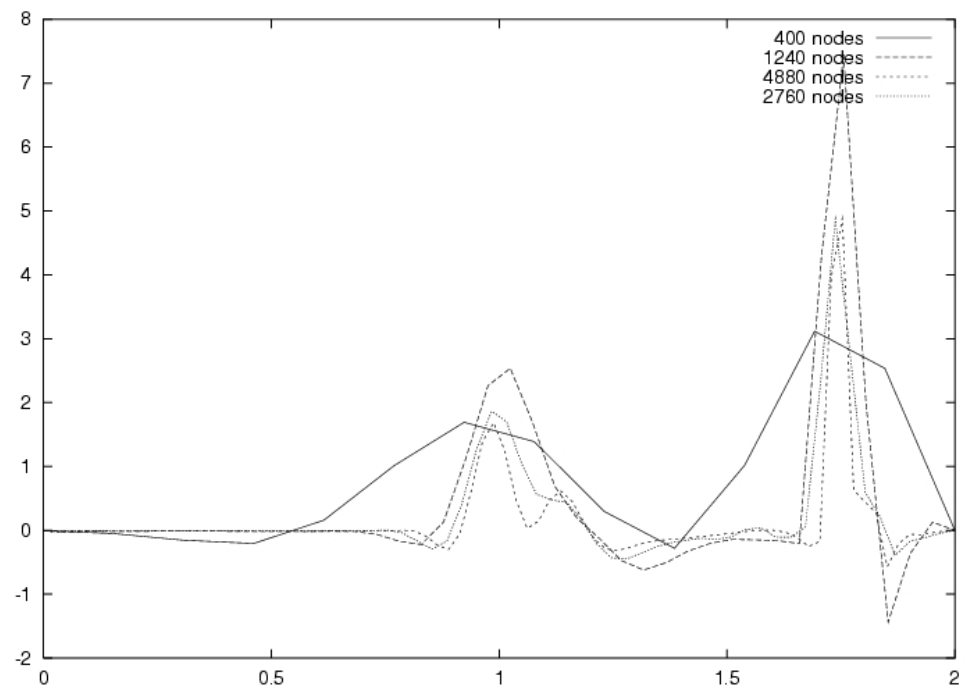

Figure 3: Optimisation d'une tuyère en transsonique.

Gradient de la fonctionnelle pour plusieurs finesses de maillages

\section{Gradients Discrets}

Une plateforme pour le design est un ensemble de logiciels permettant d'optimiser un produit à partir de simulations. Lorsque le modèle numérique est un peu complexe, et lorsque les paramètres de forme doivent être discrétisés finement, la tranformation d'un noyau de simulation en plateforme d'optimisation nécessite le développement d'un calcul de sensibilité reposant sur un état adjoint, ce que nous ferons par Différentiation Automatique Inverse (DAI), cf. $[9,11,12,12,16,17]$. Le point de départ est un logiciel de simulation contenant: - un assemblage: calculant, à partir des paramètres de design $\gamma$ et des cinq champs $\left(W_{1}\right.$ à $\left.W_{5}\right)$, le résidu des équations d'Euler ("opérateur, discret cette fois, $\Psi$ "):

$$
\text { ASSEMBLAGE-ÉTAT: }(\gamma, W) \rightarrow \Psi(\gamma, W)
$$

- un algorithme de résolution-état (appelant ASSEMBLAGE-ÉTAT):

$$
\text { RÉSOLUTION-ÉTAT: } \gamma \rightarrow W(\gamma) \text {, tel que } \Psi(\gamma, W(\gamma))=0 \text {. }
$$

- une partie post-évaluation qui calcule une fonction coût explicitement à partir du contrôle et de l'état:

$$
\text { ÉVALUATION-COÛT: }(\gamma, W) \rightarrow J(\gamma, W) \text {. }
$$

La dérivation composée d'une fonction:

$$
u \mapsto v=\Phi(u)=\phi_{p} \circ \phi_{p-1} \circ \phi_{p-2} \circ \cdots \circ \phi_{1}(u)
$$


peut se faire suivant le mode tangent:

$$
\begin{aligned}
\Phi^{\prime}(u)= & \left(\phi_{p}^{\prime} \circ \phi_{p-1} \circ \phi_{p-2} \circ \cdots \circ \phi_{1}(u)\right) \\
& \cdot\left(\phi_{p-1}^{\prime} \circ \phi_{p-2} \circ \cdots \circ \phi_{1}(u)\right) \\
& \cdot \ldots \\
& \cdot\left(\phi_{1}^{\prime}(u)\right) .
\end{aligned}
$$

Le mode tangent appliqué à la routine calculant $\Phi$ produit une routine calculant à partir de $u$ et d'une direction arbitraire $\dot{u}$ de même dimension que $u$ la dérivée dans la direction $\dot{u}$ :

$$
u, \dot{u} \mapsto \frac{\partial \Phi}{\partial u}(u) \dot{u} .
$$

La routine résultante réalise la dérivation composée dans le même ordre que la routine initiale et livre une seule donnée réelle si $\Phi$ est une fonctionnelle. La dérivation peut se faire suivant le mode inverse:

$$
\Phi^{\prime *}(x) \cdot \bar{y}=\phi_{1}^{\prime *}\left(x_{0}\right) \cdot \phi_{2}^{\prime *}\left(x_{1}\right) \ldots \phi_{p}^{\prime *}\left(x_{p-1}\right) \cdot \bar{y}
$$

Le mode inverse appliqué à la même routine calculant $\Phi$ produit une routine qui calcule à partir de $u$ et d'un vecteur arbitraire $\bar{v}$ de même dimension que $v$ le produit suivant, de même dimension que $u$ :

$$
u, \bar{v} \mapsto\left(\frac{\partial \Phi}{\partial u}(u)\right)^{*} \bar{v}
$$

Dans l'implémentation la moins complexe en calculs, les calculs se déroule d'abord dans le même sens que la routine initiale en stockant les résultats puis dans le sens inverse en utilisant les données obtenues durant la première phase. Plus généralement, les données sont soit stockées soit recalculées. Dans le cas d'une fonctionnelle dépendant de $n$ variables, la routine issue du mode inverse donne $n$ résultats et peut être $n / 2$ fois plus efficace que le mode tangent. Dans l'application qui suit, le logiciel TAPENADE , cf. [16], a été systématiquement utilisé pour la DAI d'un noyau Euler, et a permis d'assembler tous les termes du système d'optimalité:

$$
\left\{\begin{aligned}
\Psi(Y, u) & =0 \\
\left(\frac{\partial \Psi}{\partial Y}(Y, u)\right)^{*} \Pi-\left(\frac{\partial J}{\partial Y}(Y, u)\right) & =0 \\
j^{\prime}(u)=\left(\frac{\partial J}{\partial u}(Y, u)\right)-<\left(\frac{\partial \Psi}{\partial u}(Y, u)\right)^{*} \Pi, 1> & =0
\end{aligned}\right.
$$

Assembler n'est pas résoudre: les étape suivantes dans la construction de la plate-forme de design sont (a) la mise en place d'un algorithme pour résoudre l'état adjoint, puis (b) celle d'un algorithme de minimisation de $j$ : gradient ou SQP.

\section{Préconditionnement de L'optimisation}

On se propose de construire un opérateur de préconditionnement $B$ tel que l'itération suivante

$$
\gamma^{n+1}=\gamma^{n}-\rho B g\left(\gamma_{n}\right),
$$

où $g\left(\gamma_{n}\right)$ est nous l'avons vu un opérateur non borné de degré 1 , sera une itération régulière.

\subsection{Préconditionneur multiniveau additif}

Bramble, Pasciak et Xu [6] ont proposé un préconditionneur additif qui a fait l'objet de nombreuses extensions et connections notamment avec les ondelettes. L'espace continu $V$ est approché par une suite (infinie) d'espaces d'approximations emboités et de plus en plus fins:

$$
V_{0} \subset \cdots \subset V_{k} \subset \cdots \subset V .
$$


Pour fixer les idées, les sous-espaces $V_{k}$ 's sont par exemple des discrétisations sur des maillages emboités quasiuniformes, avec une taille de maille $h_{k}$ :

$$
h_{k} \approx 2^{-k}
$$

et pour tout $k$ on introduit l'opérateur de projection de fin à grossier $Q_{k}: V \longrightarrow V_{k}$ défini par:

$$
Q_{-1} u=0 \text {; et } \forall k \geq 0,\left(Q_{k} u, v\right)=(u, v) \forall v \in V_{k}
$$

Alors le préconditionneur de Bramble, Pasciak et Xu s'écrit:

$$
B_{a}=\sum_{k=1}^{\infty} 2^{-k(a+\alpha)}\left(Q_{k}-Q_{k-1}\right)
$$

où $Q_{k}$ est une projection sur $V_{k}$. $B_{a}$ est pour $\alpha>0, a>0$, un opérateur compact de degré $-a$. En pratique, on prend $\alpha=0$. $B_{0}$ est donc l'identité. Dans le cas $1 \mathrm{D}$ cartésien et périodique, $B_{-2}$ est l'inverse de l'operateur de Laplace. Dans nos applications, les équations de l'aérodynamique sont maillées sur des tétraédrisations non-structurées. La technique de construction de niveaux grossiers par agglomération de volume duaux (comme schématisé en 2D par la figure 4) permet de construire des $B_{a}$ sans déteriorer leur propriétés de préconditionneurs, cf. [8]. Cette méthode est appliquée sur le maillage de peau de l'aéronef au paramétrage de sa forme.
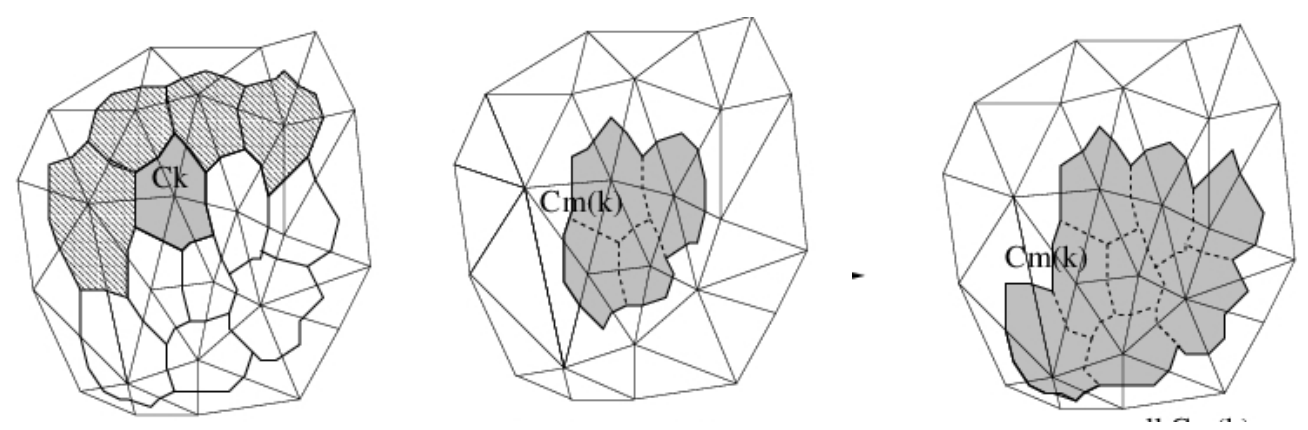

Maillage non-structuré: construction de niveaux plus grossiers par agglomération de volume.

De gauche à droite, cellule fine de niveau $k$, cellule grossère de niveau $k-1$, cellule grossère de niveau $k-2$

\subsection{Illustration numérique}

L'usage de préconditionneurs fonctionnels s'impose dans des applications aussi concrètes que l'optimisation de la voilure d'un avion. L'exemple présenté ici est tiré de [21].
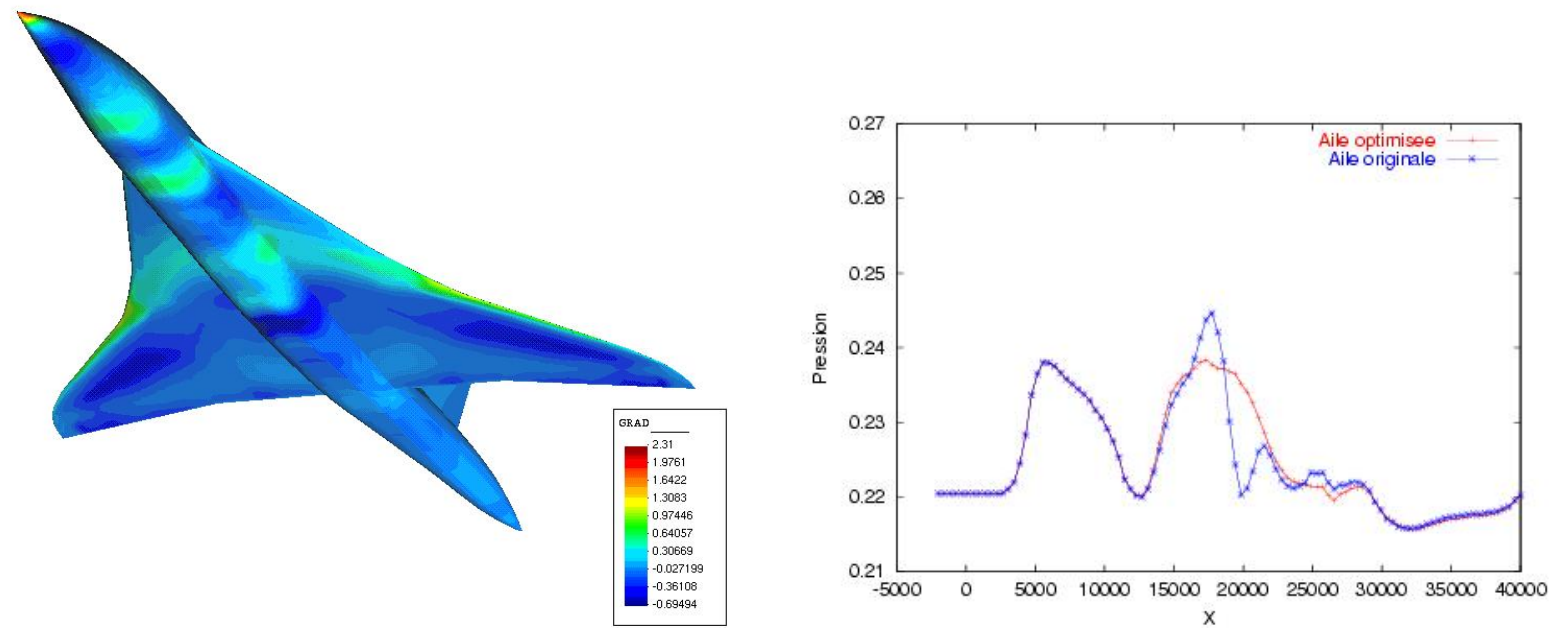
Figure 7: Optimisation du bang sonique: à gauche, visualisation du gradient de la fonctionnelle, à droite, coupe axiale de la pression dans un plan sous l'avion après 10 itérations d'optimisation

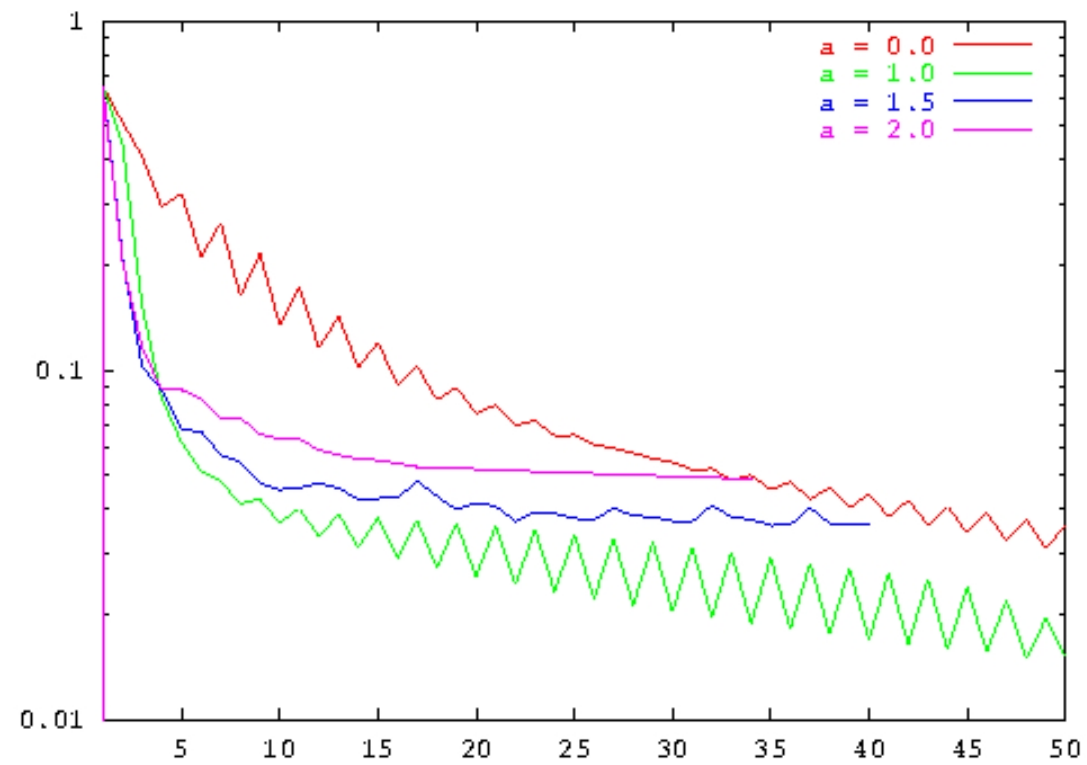

Figure 8: Application du préconditioneur $B_{a}$ avec différents $a$. Evolution de la norme du gradient.

Comme dans le paragraphe 1.2, la fonction $\gamma$ contrôle la forme de l'avion et l'équation d'état est le système des équations d'Euler tridimensionnel. L'impact du bang sonique est pris en compte (de manière très simplifiée) par une intégrale du gradient (lissé) de pression sous l'avion. Deux autres termes prennent en compte le contrôle de l'écart à des coefficients de trainée $C_{T}$ et portance $C_{P}$ cibles:

$$
j(\gamma)=\alpha_{1} \int_{\Omega^{B}}|\nabla p|^{2} d V+\alpha_{2}\left(C_{T}-C_{T}^{c i b l e}\right)^{2}+\alpha_{3}\left(C_{P}-C_{P}^{c i b l e}\right)^{2}
$$

Dans le calcul considéré la discrétisation du paramètre de forme $\gamma$ comporte 20,000 degrés de liberté, et la variable d'état $W(\gamma)$ comporte 850,000 degrés de liberté. La figure 7 montre d'une part les valeurs du gradient de forme visualisées sur le fuselage d'une géométrie SSBJ de Dassault-Aviation, et d'autre part l'effet de l'optimisation de forme sur une coupe de la pression sous l'avion. La figure 8 met en évidence l'impact sur la convergence du choix du préconditionneur adéquat. En effet, seul le préconditionneur de degré -1 permet en 10 itérations d'atteindre un niveau de norme de gradient difficilement atteint, même après 50 itérations, avec d'autres degrés de préconditionnement. De manière assez classique, le comportement des autre options s'interprêtent ainsi: les preconditionneurs de trop fort degré (plus grand en valeur absolue que l'unité) vont au début plus vite sur les basses frequences de la forme mais ensuite stagnent sur les hautes frequences car étant trop lissees, elles n'ont pu progresser. Avec un pas de gradient optimal, le cas non préconditionné (degré zéro) bute dès le début sur le lissage des hautes fréquences.

\section{Synthìse ET NOUVELLES QUESTIONS}

Calculer des gradients discrets "exacts" est de plus en plus facilité par les outils de Différentiation Automatique. Cela permet de résoudre un problème de forme optimale sur un maillage donné, supposé suffisamment fin. Mais se placer directement en dimension finie ne permet pas de comprendre et de maitriser les problèmes de régularité abordés dans ce papier. En effet il est important de connaître jusqu'à quel point la fonctionnelle continue est différentiable, et dans ce cas quelle est la régularité du candidat gradient. Néanmoins, la construction d'une méthode de gradient en continu reste un problème ouvert, dans la mesure où le point fixe n'est pas 
défini dans un contexte hilbertien. Le problème de la convergence du gradient discret vers le gradient continu reste aussi d'actualité. En effet les approches de convergence en maillage et notamment celles reposant sur des maillages adaptatifs ne permettent pas de conserver une seule fonctionnelle discrète à minimiser. Il devient dans ce cas nécessaire d'appréhender la convergence en maillage comme un processus qui concerne les trois équations du système d'optimalité et donc le gradient.

\section{REFERENCES}

[1] W. K. Anderson and V. Venkatakrishnan. Aerodynamic design optimization on unstructured grids with a continuous adjoint formulation. Computers and Fluids, 28(4).

[2] E. Arian and M.D. Salas. Admitting the inadmissible: Adjoint formulation for incomplete cost functionals in aerodynamic optimization. Research report 69, Institute for Computer Applications in Science and Engineering (ICASE), 1997.

[3] C. Bardos and O. Pironneau. A formalism for the differentiation of conservation laws. C. R. Acad. Sci. Paris, 335(10):839, 2000.

[4] C. Bardos and O. Pironneau. Derivatives and control in the presence of shocks. CFD J., 12(1), 2003.

[5] F. Beux and A. Dervieux. Exact-gradient shape optimization for a 2-D Euler flow. Finite Elements in Analysis and Design, 12:281-302, 1992.

[6] J. Bramble, J. Pasciak, and J. Xu. Parallel multilevel preconditioners. Math. Comput., 55(191):1-22, 1990.

[7] J. Céa. Conception optimale ou identification de forme. Calcul rapide de la dérivée directionnelle de la fonction coût. $R A I R O$ Modél. Math. Anal. Numér., 20:371-402, 1986.

[8] F. Courty and A. Dervieux. Multilevel functional Preconditioning for shape optimisation. Int. J. CFD, 20(7):481-490, 2006.

[9] F. Courty, A. Dervieux, B. Koobus, and L. Hascoët. Reverse automatic differentiation for optimum design: from adjoint state assembly to gradient computation. Optimization Methods and Software, 18(5):615-627, 2003.

[10] A. Dervieux. Perturbation des équations d'équilibre d'un plasma confiné: comportement de la frontière libre, étude des branches de solutions. Research Report 10, INRIA, 1980.

[11] A. Dervieux, F. Courty, T. Roy, M. Vázquez, and B. Koobus. Optimization loops for shape and error control. In PROMUVAL Short Course on Multidisciplinary Modelling, Simulation and Validation in Aeronautics, Barcelona, june 28-29, 2004. CIMNE, 2004. extended version: INRIA Research Report 5413.

[12] M. Giles. Using Automatic Differentiation for adjoint CFD code development. In Post-SAROD workshop, Bangalore, India, 2005.

[13] M.B. Giles and N.A. Pierce. On the properties of solutions of the adjoint euler equations. In Numerical Methods for Fluid Dynamics VI, ICFD, Oxford, 1998.

[14] M.B. Giles and N.A. Pierce. An introduction to the adjoint approach. to design. In ERCOFTAC Workshop on Adjoint Methods, Toulouse, 1999. June 21-23.

[15] M.B. Giles and N.A. Pierce. Analytic adjoint solutions for the quasi-1D Euler equations. J. Fluid Mech., $426: 327,2001$.

[16] L. Hascoët and V. Pascual. Tapenade 2.1 user's guide. Technical Report 0300, INRIA, 2004.

[17] L. Hascoët, M. Vázquez, and A. Dervieux. Automatic differentiation for optimum design, applied to sonic boom reduction. In V.Kumar et al., editor, Proceedings of the International Conference on Computational Science and its Applications, ICCSA'03, Montreal, Canada, pages 85-94. LNCS 2668, Springer, 2003.

[18] C. Homescu and I.M. Navon. Optimal control of flow with discontinuities. Journal of Computational Physics, 187(2):660 $682,2003$.

[19] S. Nadarajah and A. Jameson. A comparison of the continuous and discrete adjoint approach to automatic aerodynamic optimization. In AIAA 38th Aerospace Sciences Meeting and Exhibit, AIAA-2000-0667, Reno, NV, 2000.

[20] S. Nadarajah and A. Jameson. Studies of the continuous and discrete adjoint approaches to viscous automatic aerodynamic shape optimization. In AIAA 15th Computational Fluid Dynamics Conference, AIAA-2001-2530, Anaheim, CA, 2001.

[21] M. Vázquez, B. Koobus, and A. Dervieux. Multilevel optimization of a supersonic aircraft. Finite Element in Analysis and Design, 40:2101-2124, 2004. 